\title{
Occlusal plane related to skeletal pattern in mixed dentition stage: a descriptive cephalometric study
}

\author{
S. Naretto, C. Polastri, R. Slavicek \\ Department of Interdisciplinary dentistry and Technology, Danube University, Krems, Austria
}

Received November 29, 2008; Accepted December 20, 2008

Basic studies and researches on craniofacial growth show that occlusal plane changes its position in space and time during the whole period of development and growth of the skull until the attainment of the mature dentition stage. The purpose of this retrospective study is to evaluate the inclination of the gnatological occlusal plane related to the axio-orbital plane and their relations with skeletal pattern in a group of subjects during the period of mixed dentition stage. This is performed analyzing cephalometric tracings of 714 untreated children between 5.6 and 12 years old. Observations of data indicate that there is difference in inclination between skeletal class I, class II and class III, being more steep in class II and more flat in class III. Variations between the "subclasses" demonstrate very high degree of "individuality" of the inclination of the occlusal plane related to the functional axio-orbital plane in subjects in mixed dentition stage.

Keywords: Occlusal plane, mixed dentition, skeletal pattern, cephalometrics, descriptive study

\section{Introduction}

The occlusal plane, one of the important factors for the morphology and the function of the masticatory organ, is a simple way to visualize skeletal and occlusal relationships. It is a bidimensional description of the sum of the occlusal planes of each single tooth, therefore is a pure functional plane.

The shape and inclination of the occlusal plane is an individual characteristic and is connected with the function of the stomatognathic system [1]. Dynamic changes in the craniofacial growth effect many changes in occlusal relationships: the occlusal plane modifies its position in space during the whole period of development of the skull until the attainment of the mature dentition stage. The final position idepends on several factors related to the biomechanical behaviour during functions of the masticatory organ.

Previous studies based on cephalometric measurements have been published evaluating the relation between occlusal

Correspondence: Carolina Polastri, Department of Interdisciplinary dentistry and Technology, Danube University, Dr.-Karl-Dorrek-Straße 30, 3500 Krems, Austria.

E-mail: carolinapolastri@gmail.com plane and skeletal patterns [1, 5-14, 16, 17]. Different authors $[5,6,13]$ pointed out that mandibular rotation during growth results from dissimilar increments of vertical growth between the mandibular condyle and fossa on the one hand, and the maxillary sutures-alveolar processes on the other. The occlusal plane is the effect, not the cause, of anatomic relationships attributing condylar growth (related to vertical growth) as the key to the changes of the occlusal plane.

In a metallic implant study, Bjork [2] and Bjork and Skieller $[3,4]$ showed that maxillary growth was not merely characterized by anterior-inferior displacement, but also a forward rotation accompanied by descent of the upper molar region and a simultaneous forward mandibular rotation. In other words, the rotational growth of the maxilla causes a flattening of the occlusal plane in the upper dentition, and the mandible grows anterior-inferiorly with a forward rotation maintaining occlusal contacts with the upper dentition.

Vukusic [17] examined the change in the occlusal plane inclination that takes place during craniofacial growth relative to various facio-cranial reference lines. The investigation was carried out on 192 lateral radiographs of subjects divided into five age groups that ranged from 10 to 18 year olds. He concluded that the change in occlusal plane inclination shows anterior rotation (left profile) during growth. There were no significant differences according to sex.

In another study Tamaki et al. [16], using cephalometric measurements of 117 subjects from 18 to 39 year olds, pointed out that there were significant correlations between the inclination of the occlusal plane to the axio-orbital plane and the APDI [8] (anteroposterior displasia indicator) and between the vertical dimension and the APDI.

Aim of the study is to evaluate the inclination of the gnatological occlusal plane by definition related to the axioorbital plane as functional reference plane in a group of subjects during the period of mixed dentition stage. The inclination of the occlusal plane was correlated to skeletal.

pattern referring to the classification purposed in a basic research published from Prof. Dr. Rudolf Slavicek [15]. Measurement of the inclination of the occlusal plane can be used to predict the posterior-anterior position of the mandible related to skeletal pattern. During mixed dentition stages of growth, the inclination of the occlusal plane can be orthodon- 
tically controlled to avoid further development of skeletal disgnatia and severe malocclusion.

\section{Material and methods}

The retrospective study considered lateral X-ray data, of a group of 714 European children, collected in the past four years. Patients, males and females, of age between 5.6 and 12.0 years, were orthodontically untreated, in an average dental status defined as good, and without any particular dental intervention, extractions or important dental treatments.

Cephalometric tracing was realized with the CADIAS software from Gamma dental software version 5.1.1.0.

The software is able to evaluate the inclination of the gnatological occlusal plane by definition, the plane designed between the edge of the mandibular incisors and the distal cusps of the two mandibular molars, related to the axio-orbital plane.

Classification of skeletal pattern was performed by the software considering skeletal classes I, II and III divided into subclasses in relation to the basic research of the author [15].

The statistical analysis was performed with the software Lab-Stat 5.0, StatSoft Inc.

The whole group was classified first in the current skeletal classification and the results are as shown in Fig. 1, so in similar percentage as reported in the previous study [15] in a mature dentition group.

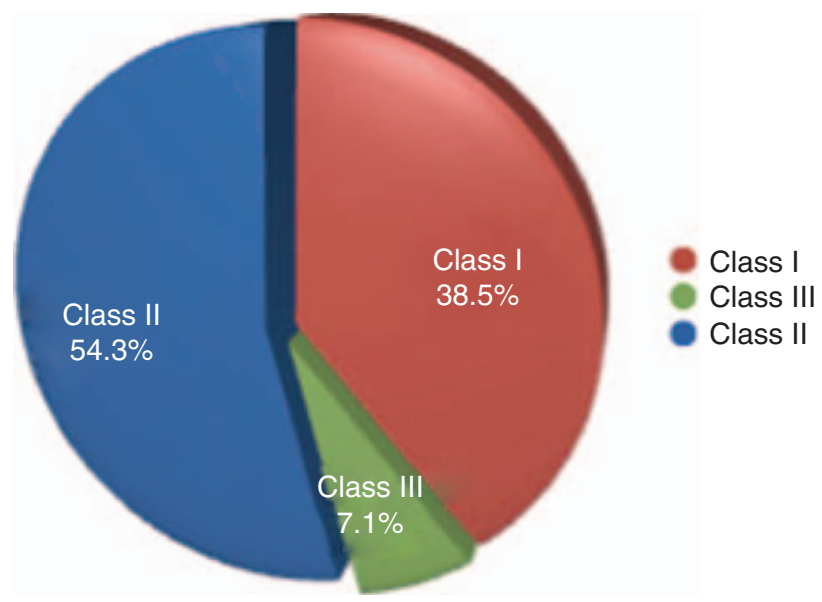

Fig. 1: Percentage division of the total sample in class I, class II and class III

\section{Tab. 1. Subclasses classification: legenda}

\begin{tabular}{ll}
\hline ER & extremely retrognatic \\
\hline SR & strongly retrognatic \\
\hline R & retrognatic \\
\hline $\mathrm{N}$ & neutral \\
\hline $\mathrm{P}$ & prognatic \\
\hline $\mathrm{SP}$ & strongly prognatic \\
\hline EP & extremely prognatic \\
\hline
\end{tabular}

The further subdivision of class I, class II and class III, referring to the relation between Maxilla and Mandible as indicated by the authors, was done using the terminology

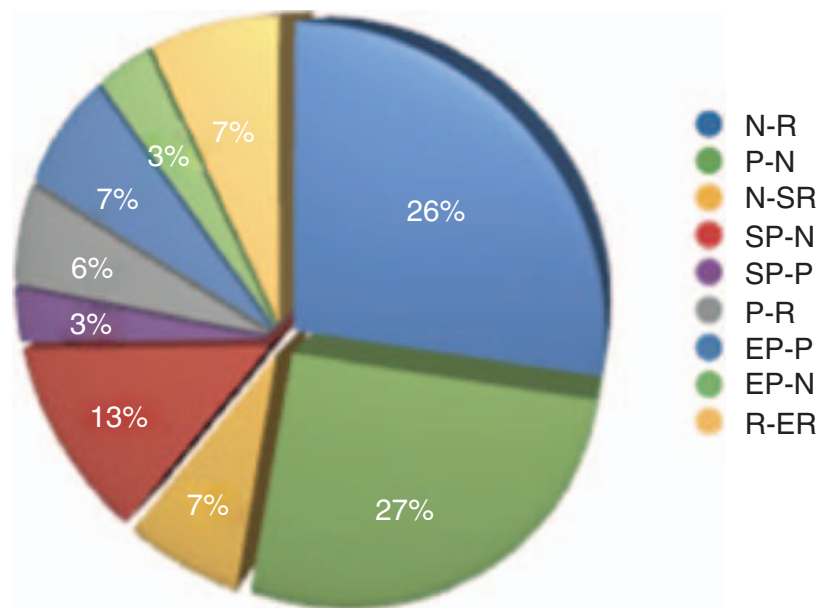

Fig. 2: Percentage division of the class II subclasses

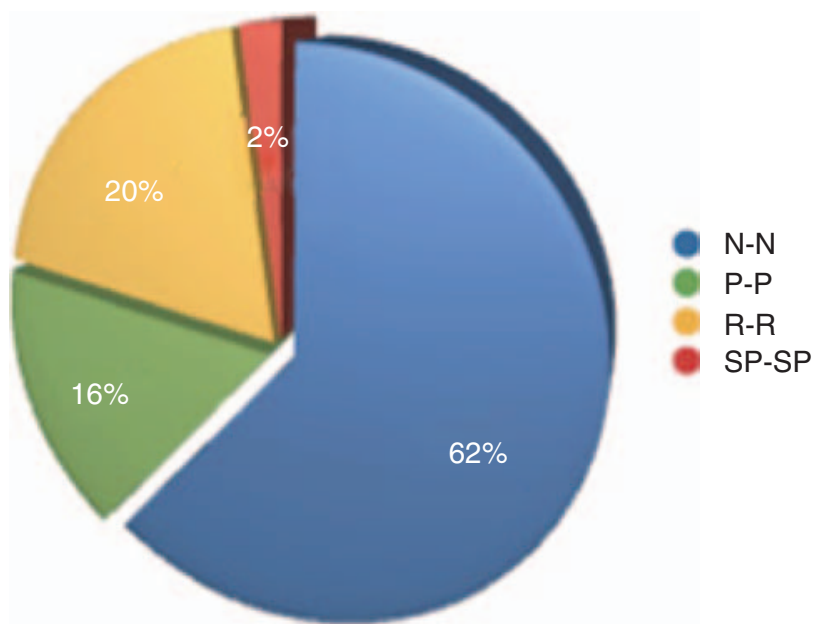

Fig. 3: Percentage division of the class I subclasses

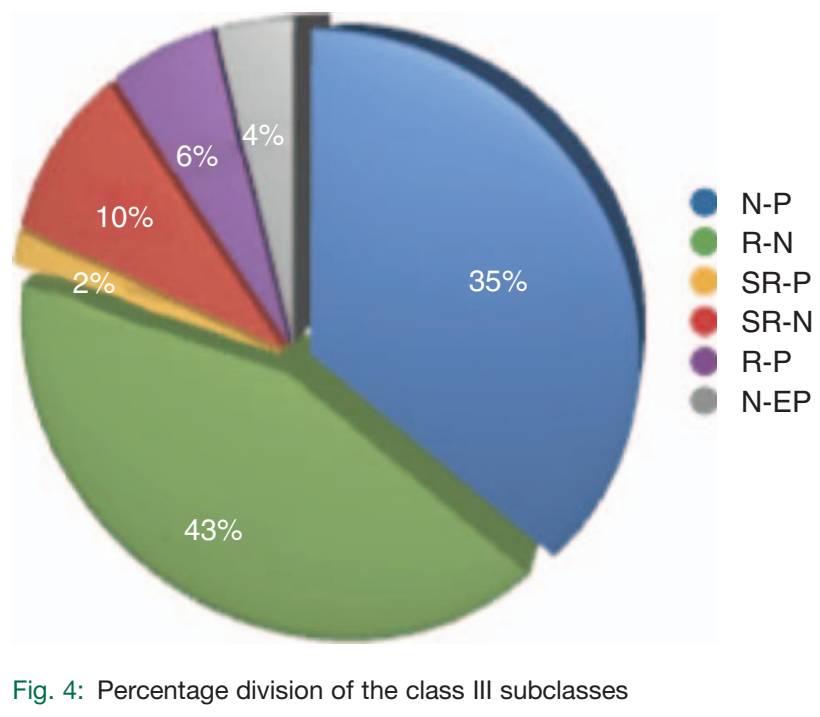


neutral, prognatic and retrognatic and present the following behaviour (Tab. 1) (Figs. 2-4).

\section{Results}

Inclination of Occlusal Plane on Axio-Orbital Plane in skeletal classes I, II, III.

Analyzing the results of the data collected it can be observed that the average inclination of the Occlusal Plane related to the Axio-orbital Plane in the total group is: $X=14.2$ with a standard deviation of 5.7 (Fig. 5). For the skeletal class I group is $X=13.5$, with a standard deviation of 5.7 (Fig. 6); for the skeletal class II is $X=15.1$ with a standard deviation of 5.7 (Fig. 7); for the skeletal class III is $X=11.3$ with a standard deviation of 4.7 (Fig. 8).

Inclination of occlusal plane on axio-orbital plane in subdivision skeletal groups.

Analyzing the data collected of subject in skeletal class it is possible to observe a variation between subclasses with a range of inclination from $12.4^{\circ}$ to $15.3^{\circ}$.

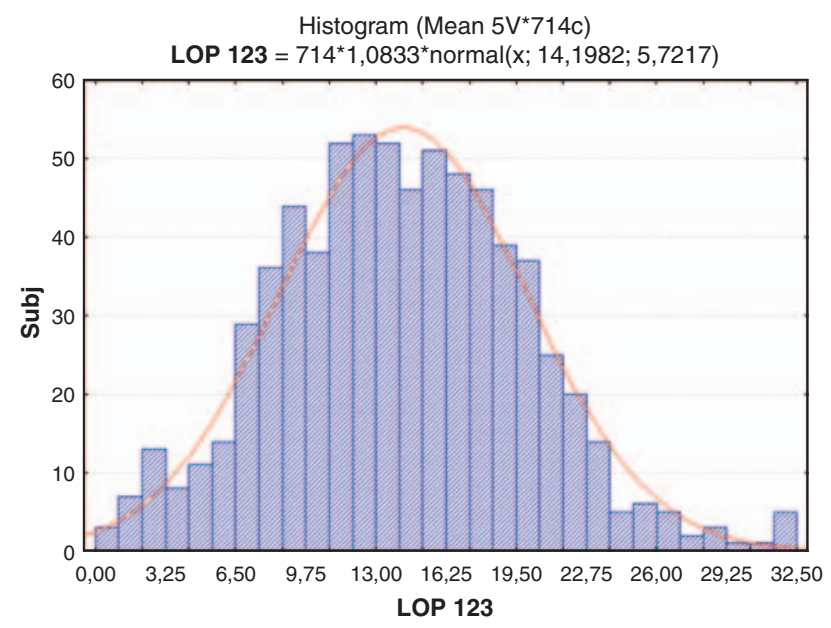

Fig. 5: Distribution of Lower occlusal plane values $\left(^{\circ}\right)$ in the total sample group, class I plus class II plus class III

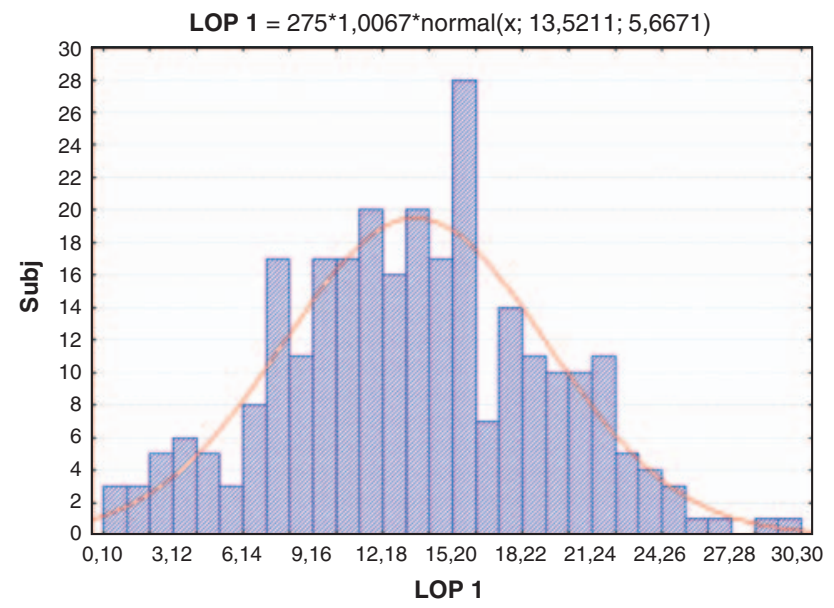

Fig. 6: Distribution of Lower occlusal plane values $\left({ }^{\circ}\right)$ in class I

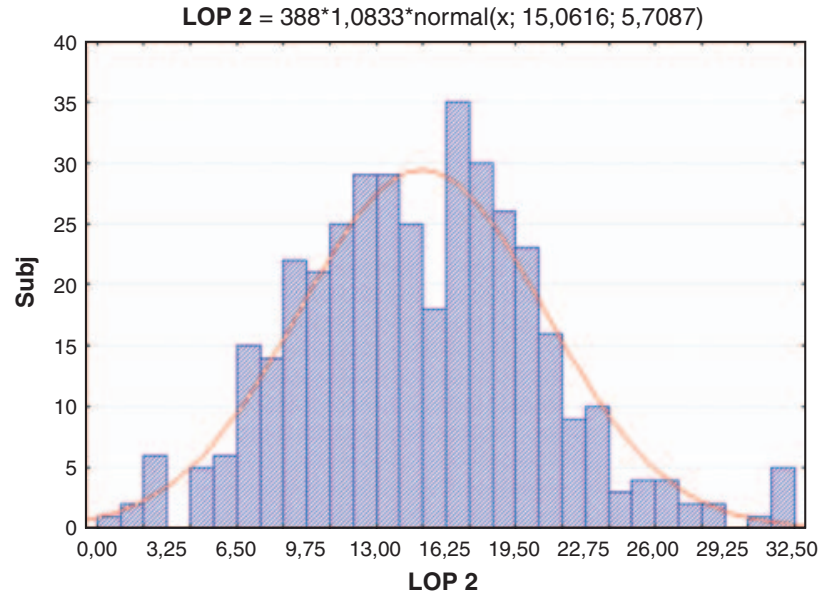

Fig. 7: Distribution of Lower occlusal plane values $\left(^{\circ}\right)$ in class II

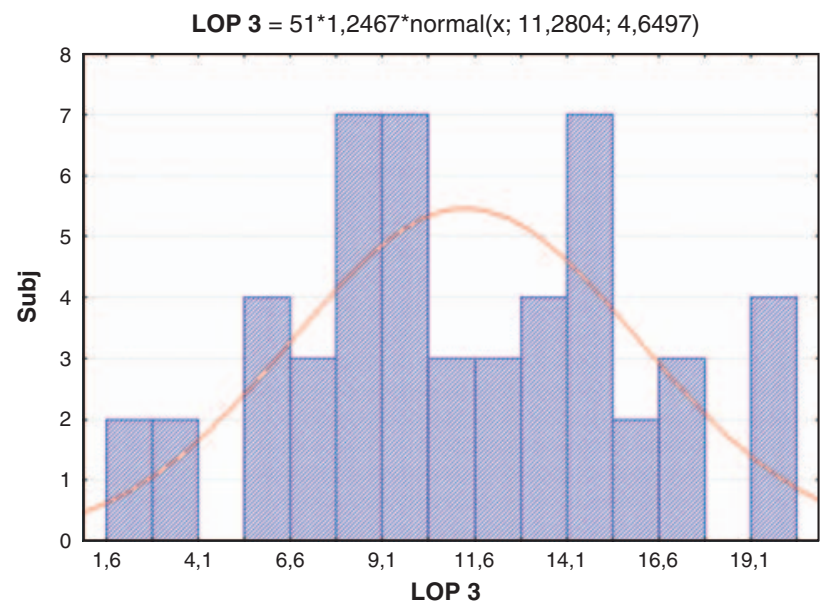

Fig. 8: Distribution of Lower occlusal plane values $\left(^{\circ}\right)$ in class III

In skeletal class II the variation is between $12.4^{\circ}$ and $18.5^{\circ}$ depending from the relation of the maxilla to the mandible.

In skeletal class III the variation between subclasses is between $4.7^{\circ}$ and $13.4^{\circ}$.

Observing the final data graph (Fig. 9), it comes out that the inclination of the occlusal plane has a wide range of variability.

\section{Discussion and conclusion}

As already indicated from the previous study [16], the difference in inclination is between skeletal class I, class II and class III, being more steep in class II and more flat in class III; this is confirming all basic study in craniofacial growth. Furthermore, we wanted to analyze the differences between subclasses in each skeletal class. We can observe that in class I the inclination is stable but in class II and class III the variability is very high.

Observing the final graph it seems to understand a progression of the value of the inclination of the occlusal plane from class II through class I and finally to class III. The study confirms that the sagittal relation between maxilla and mandible is determined by vertical growth changes and it can 


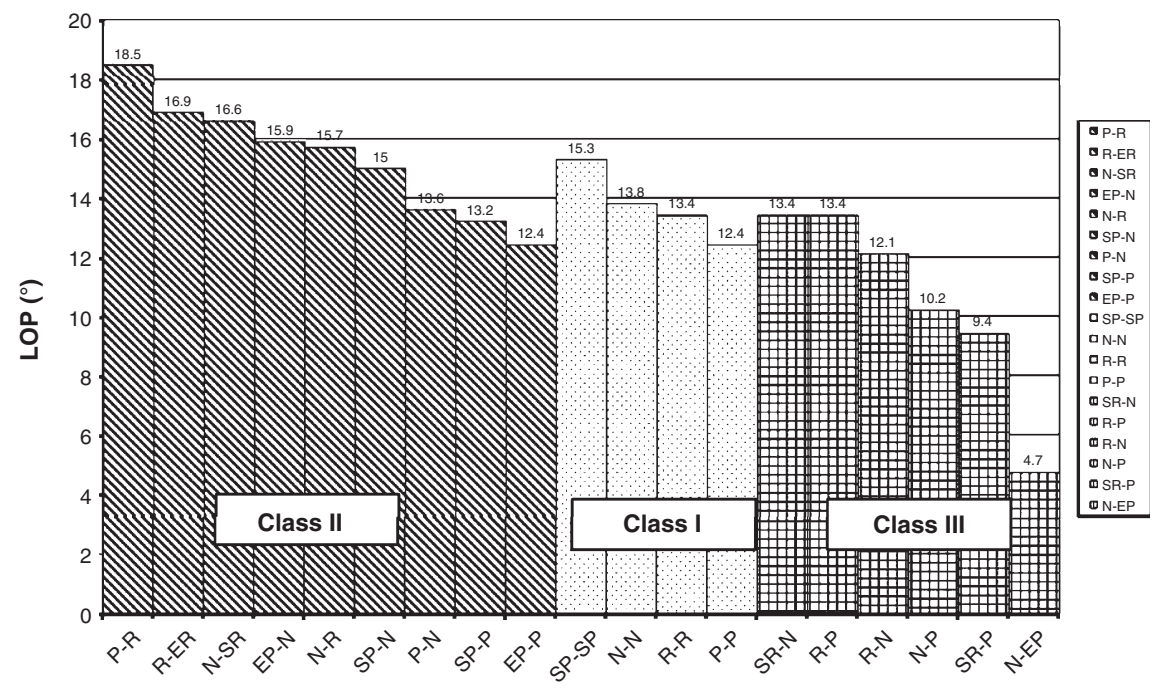

Subclasses

Fig. 9: Occlusal plane value variation within the subclasses of total sample divided in class I, class II and class III

be observed in the behaviour of variations of inclination of the occlusal plane. In conclusion, it is not correct to establish an average inclination of the occlusal plane in all subjects; also the inclination of the occlusal plane related to skeletal classes is insufficient; variations between the "subclasses" demonstrate the very high degree of "individuality" of the inclination of the occlusal plane related to the functional axio-orbital plane.

\section{References}

[1] Augsburger RH. Occlusal plane relation to facial types. J Prosthet Dent 1953;3:755-70.

[2] Bjork A. Facial growth rotation - reflections on definition and cause. Proc Finnish Dent Soc 1991;87:51-8.

[3] Bjork A, Skieller V. Facial development and tooth eruption. An implant study at the age of puberty. Am J Orthod 1972;62:339-83.

[4] Bjork A, Skieller V. Postnatal growth and development of the maxillary complex. In: McNamara JA Jr (ed.) Factors affecting the growth of the midface, Monograph 6, Craniofacial Growth Series. Center for Human Growth and Development; 1976. pp. 61-99.

[5] Creekmore T. Inibition and stimulation of vertical growth of the facial complex: its significance to treatment. Angle Orthod 1967;37: 285-97.

[6] Isaacson R, Zappel R, Worms F, Bevis R, Speidel TM. The effects of mandibular growth of the dental occlusion and profile. Angle Orthod 1977;47:97-106.
[7] Ismail YH, Bowman JF. Position of the occlusal plane in natural and artificial teeth. J Prosthet Dent 1956;20:407-11.

[8] Kim YH. Anteroposterior Dysplasia Indicator. An adjunct to cephalometric differential diagnosis. Am J Orthod 1978;73:619-633.

[9] Komiyama T, Kusuzawa O, Shimizu T, Ukiya M. Cephalometric evaluation on esthblishment of upper occlusal plane. Bull Kanagava Dent Coll 1974;2:73-8.

[10] Okuda T, Ishii $H$, Ishigaki S, Akanishi M, Maruyama T. A roentogengraphic cephalometric study on the relationship of dentofacial morphology and occlusal plane. J Jpn Prosthdont Soc 1988;32:1268-74.

[11] Pancherz H, Ruf S, Kohlhas P. Effective condylar growth and chin position changes in herbst treatment: a cephalometric roentogengraphic long-term study. AJODO, Oct 1998;114:437-46.

[12] Reitz PV, Aoki H, Yoshioka M, Uehara J, Kubota Y. A cephalometric study of tooth position as related to facial structure in profiles of human beings: a comparison of Japanese (Oriental) and American (Caucasion) adults. J Prosthet Dent 1973;29:157-66.

[13] Schudy FF. The rotation of the mandible resulting from growth: its implications in orthodontic treatment. Angle Orthod 1965;35:36-50.

[14] Skieller V, Bjork A, Linde-Hanse T. Prediction of mandibular growth rotation evaluated from a longitudinal implant sample. AJODO 1984;86(5):359-70

[15] Slavicek R. Die Funktionelle Determinanten des Kauorgans Habilitationsschrift aus Universitatsklinik. Wien; 1984.

[16] Tamaki K, Yoshino T, Celar AG. Freudenthaler J.W, Slavicek R, Sato S. A Cephalometric Study of the Compensation for the Skeletal Pattern of Dentulous Subjects. Bull Kanagava Dent 1999;27: 8-12.

[17] Vukusic N, Lapter M, Muretic Z. Change in the inclination of the occlusal plane during craniofacial growth and development. Coll Antropol Jun 2000;24(1):145-50. 